\title{
Marker based genetic variability analysis of rice (Oryza sativa L.) landraces for drought tolerance
}

\author{
Sujan Acharjee ${ }^{1}$, Nihar Ranjan Chakraborty ${ }^{2 *}$ and Sankar Prashad Das ${ }^{3}$ \\ 1,2- Department of Genetics and Plant Breeding, Institute of Agriculture, Palli Sikhsha \\ Bhavan, Visva Bharati, Bolpur, West Bengal-731236, India. \\ 3-Indian Council of Agriculture Research-Complex for North Eastern Hilly region, Tripura \\ Centre, Lembucherra, Tripura-799210, India \\ *Corresponding author e-mail: nrchakraborty@gmail.com
}

Received: February 13, 2021; Accepted:March 15,3021.; Available online: March 22, 2021.

\begin{abstract}
Under the present study, assessment of genetic variability of 60 numbers of rice landraces was carried out under morphological and molecular level for selection of promising drought tolerant genotypes under upland ecosystem of North East India. Predominance of additive gene action on characters like leaf rolling index, root: culm ratio, grain yield and other yield contributing traits like plant height came out as effective parameters for selection of drought stress tolerant landraces. Molecular analysis revealed moderate genetic diversity with average Polymorphic Information Content values of 0.44 across the rice landraces. No specific clustering patterns of landraces against some morphological traits numbers of productive tillers per plant, numbers of filled grains per plant etc were found. This signifies the polygenic nature of the quantitative characters and influence of environment on them. Precise correlation between the morphological performance of the landraces and their clustering pattern under molecular analysis was found to be effective in identifying suitable landraces like Chikanswarikabar as a promising parent for future breeding programme and also to formulate efficient strategies for sustainable management of rice landraces under rain fed ecosystem.
\end{abstract}

Keywords: Rice, Landraces, variability, drought, molecular marker. \section{INTRODUCTION}

Rice (Oryza sativa L) is the most staple food of more than half of the world's population (Rasheed et al., 2020) and $90 \%$ of it is being produced and consumed in Asia (Fukagawa and Ziska, 2019) and share maximum in grain production. Being a semi aquatic crop, rice can be grown in diverse range of ecosystems such as rain fed uplands and lowlands, irrigated, and deep-water ecosystems. But, in present day scenario, the entire ecosystems suffer from the threat of the climate change like drought stress and to be more specifically, the rain fed uplands are the most severely affected by drought stress because of its lighter soil texture and quick percolation tendency of rain water. Widespread drought occurrence in rice-growing areas results in severe decline in yield and thus the development of drought-tolerant varieties that maintain good yield under moisture stress is a priority area of rice research for sustainable rice production and improvement. The success of a crop improvement programme depends on the availability of genetic variability in the population. Rice landraces, because of their effectual evolution process and enormous in-built genetic variability can grow in a wide range of ecosystem and climatic conditions and can be treated as promising source towards development of drought tolerant population.

India is considered to be one of the prime centres for rice diversity and Tripura, being a state of north east India is endowed with a great diversity of rice landraces in its varied topographical rain fed upland ecosystem. Many of the rice landraces grown in the hilly region of this state are found to be effective as sources of drought tolerance. But, over the last few 
Sujan Acharjee et al.

decades, rice has faced a severe diversity loss like other locally available landraces of different crop plants that are often ignored in existence of high-yielding varieties, however, have survived environmental turmoil over the ages (Pradhan et al., 2020). Along with, it is also undoubtedly proven fact that, information on the amount of genetic variation in the germplasm and genetic relationships between them is imperative for the study and designing of breeding programs (Farahani et al., 2019) towards identification and development of germplasm and defining the existence of varieties (Eid, 2019). In any breeding programs, determination of the level of genetic diversity in crop species is of great importance for the selection of suitable parents and maximum utilization of heterosis (Luo et al., 2019). Therefore, the present study was undertaken with the aim to assess the trend in genetic diversity in sixty numbers of rice landraces under both morphological and molecular level and to generate comparative information for selection of appropriate drought tolerant promising genotypes in future rice breeding programme.

\section{MATERIALS AND METHODS Field experiment}

The field experiment was conducted in the farm complex of Indian Council of Agricultural Research (ICAR) Complex for North Eastern Hilly Region, Tripura Centre, Lembucherra, West Tripura $\left(23^{\circ} 90^{\prime} \mathrm{E}, 92^{\circ} 29^{\prime} \mathrm{N}\right)$, India, during the years 2015-16 and 2016-17. 60 numbers of local rice land races (Table1), collected from different hill ranges of Tripura were evaluated through Randomised Complete Block Design with 3 replications. Artificial drought conditions were imposed in the experiment field during the 'Boro' seasons of 2015-16 and 2016-17 as per the standard procedure of IRRI Knowledge bank (http://www. knowledgebank.irri.org/rice breeding course). Seeds were directly sowed at a distance of $25 \mathrm{~cm}$ (row-row) x $25 \mathrm{~cm}$ (plant -plant) in dry soil. Regular furrow type of irrigation has been provided up to 30 days after sowing to ascertain optimum seedling growth. After that, the frequencies of irrigation were reduced. No irrigation was provided until or unless field surface is completely dry or plants under the study showed severe wilting symptoms. When the target level of soil dryness and plant stress are reached, the field was liberally irrigated to saturate the root zone. This irrigation pattern was repeated until harvest. Fertilizers were applied @ 60:40:40 Kg N: P: $\mathrm{K} \mathrm{ha}^{-1}$. The same pattern of management practices, irrigation schedules, inter culture operation other pest control measures were followed as and when necessary during the whole growing periods for the investigated years.

\section{Phenotypic analysis based on morphological traits}

Pulled mean values of 15 numbers of morphological characters as listed in Table (2) were recorded from five numbers of competitive plants preferably from the middle rows over the replications. Standard evaluation system of Rice (IRRI, 2002) was considered as reference while recording the characters. The mean data were also subjected to heritability and genetic advance analysis as per Johnson et al. (1955) using Windostat Version 9.2 from Indostat service. In order to arrange the landraces in various groups and subgroups and also to find out the behavioural similarity of them, Hierarchical cluster analysis for all the morphological traits in 60 rice land races was carried out using un-weighted pair group linkage type through NCSS statistical software 2019. The Euclidean distance method and standard deviation scaling method were used with cluster cut off value of 1.0 to form a horizontal dendrogram.

\section{Molecular analysis}

DNA was extracted from the tender leaf tissues of 21 days old seedlings (a 


\section{Marker based genetic variability analysis of rice (Oryza sativa $L_{\text {. }}$ ) landraces for drought tolerance}

healthy single seedling per genotype) of the rice land races, based on Cetyl trimethyl ammonium bromide (CTAB) method described by Muray and Thompson (1980) with some modification. A set of 40 numbers of simple sequence repeat (SSR) markers, distributed among different rice chromosomes were screened and subsequently utilised for genetic diversity analysis of the rice land races. Polymerase chain reactions were carried out in $12.5 \mu \mathrm{l}$ of total reaction volume containing $1.0 \mu \mathrm{l}$ genomic DNA , $4.25 \mu \mathrm{l}$ nano pure $\mathrm{H}_{2} \mathrm{O}, 6.25 \mu \mathrm{l}$ of PCR master mix (Hi Media) containing Thermus aquaticus (Taq) DNA polymerase, dNTPs, $\mathrm{MgCl}_{2}$ and reaction buffers at optimal concentrations and $0.5 \mu \mathrm{l}$ from microsatellite (SSR) markers (forward and reverse primers). Amplifications were performed in Eppendorf nexus gradient Master cycler with the temperature profile consisting of Initial denaturation stage of $94^{\circ} \mathrm{C}$ for 5 minutes and 35 cycles of [(i) Denaturation stage : $94^{\circ} \mathrm{C}$ for 1 minute (ii) Annealing stage : $55^{\circ} \mathrm{C}$ for 45 seconds ( iii Extension stage : $72^{\circ} \mathrm{C}$ for 2 minutes ] followed by final extension stage of $72^{\circ} \mathrm{C}$ for 10 minutes. The ultimate PCR products were then diluted in $50 \mu \mathrm{l}$ of distilled water and kept in $4^{\circ} \mathrm{C}$ for further $\mathrm{Gel}$ electrophoresis. Subsequently, around 2 $\mu \mathrm{L}$ aliquot of amplified PCR products of each of the landraces were mixed gently with loading buffer and loaded in $2 \%$ agarose gel prepared in Tris- Acetate (TAE) buffer along with $100 \mathrm{bp}$ and $50 \mathrm{bp}$ DNA marker ladder. For gel electrophoresis of SSR amplified PCR products, agarose gel were also used by several previous workers (Anupam et al.,

2017; Ravikiran et al., 2018; Hamidah et al., 2020) with resultant significant polymorphism. The amplified PCR products were then size fractioned by electrophoresis and visualized by staining with ethidium bromide $(0.5 \mu \mathrm{g} / \mathrm{ml})$ in MultiDoc-It Imaging System of UVP.
For diversity analysis at molecular level, only consistent and reproducible bands were scored in binary format as ' 1 ' for presence and ' 0 ' for absence. Smeared and weak bands were excluded. Fragments of the same molecular weight were considered to represent the same allele. Polymorphic information content (PIC) is a measure of a marker's ability to detect polymorphism in a population, based on the number of alleles detected and their frequency distribution; hence, it provides an estimate of the discriminating power of a marker.PIC is calculated for each marker using the formula: $\mathrm{PIC}_{\mathrm{i}}=1-\Sigma \mathrm{P}^{2} \mathrm{ij}$, where $\mathrm{Pij}$ is the frequency of the $\mathrm{jth}$ allele in genotype (i). The polymorphism information content for each locus and heterozygosity was calculated using PIC calculator (Jan, 2002). Di-nucleotide repeat motifs, expected amplified band sizes and annealing temperatures of microsatellite primers under study were availed from SSR marker resources of Gramene microsat database (https://archive.gramene.org/markers/micr osat/all-ssr.html), whereas, major allele frequency of a specific primer was calculated out considering the numbers of occurrence of that particular allele and total numbers of alleles. SSR allelic binary ( 0 and 1) data generated for 60 rice land races was converted to text tab limited format and imported to DARwin software VERSION 6 (Perrier and JacquemoudCollet, 2006). Dissimilarities of allelic data were calculated with 1000 bootstraps option using simple matching dissimilarity index. Unweighted Neighbour joining method was adopted while constructing the horizontal dendrogram based on the allelic dissimilarity.

RESULTS AND DISCUSSION Phenotypic analysis based on morphophysiological traits:

Under the study, the differences between the phenotypic coefficient of variations (PCV) and genotypic coefficient of variations (GCV) for some of the 


\section{Sujan Acharjee et al.}

studied characters were found to be comparatively thin indicating sensitivity to environment and consequently greater role of drought stress influencing the expression of those characters. The magnitude of PCV and GCV were found to be highest in case of leaf rolling Index (LRI) followed by leaf tip drying index (LDI). High percentile of coefficient of variations values of LRI and LDI are in agreement with the research works of Haider et al. (2012) and Kumar et al. (2015).

High heritability coupled with moderate to high genetic advance have been observed in LRI, Root: culm ratio, numbers of filled grain per panicle, numbers of spikelet per panicle, grain yield per plant and 100 seed weight which implies predominance of additive gene action for those character. Thus, selection of land races based on those characters would be effective. Similar types of findings under drought stress condition have also been reported by Haider et al. (2012) for LRI, Panja et al. (2017) for root:culm ratio, and Perween et al.(2020) for numbers of filled grains per panicle. High value of heritability along with moderate to high magnitude of genetic advance of traits viz. numbers of spikelet per panicle and grain yield and test weight were also observed by Perween et al. (2020) and Nithya et al. (2020). The estimates of GCV, PCV, Heritability and Genetic advance etc are presented in Table (3).

For effective selection of promising rice landraces, grouping of the land races had also been carried out based on the mean value analysis of some significant morphological traits on the basis of Standard evaluation system of Rice, IRRI 2002 (Table 4), wherein, landraces namely ChikanswariKabar, Garo Malati, Galong, Turkey, Tarkol, Dhalabalam, Maimi Hungar were found to be better performer as far as the grain yield under moisture stress condition is considered. Thus, those landraces may be opted as parent materials for subsequent breeding programme.

\section{Molecular analysis based on} microsatellite-based rice marker:

Out of 40 micro satellite or SSR primers, 39 numbers of primers generated polymorphic alleles, while only one marker (RM 172) produced monomorphic allele. A total number of 119 alleles were detected at the loci of the 40 numbers of SSR markers across the sixty rice landraces with an average count of 2.97 alleles per locus. This is an indication of moderate level of molecular diversity among the landraces under the study.

The highest numbers of total alleles per locus were observed for RM252 (6), RM254 (5), followed by RM505, RM285, RM320, RM175, RM339, RM276 and RM592 with 4 numbers of alleles per locus for each primer. The result is confirmatory with the studies of Yadav et al. (2013) against RM252 and Anupam et al. (2017) against RM252 and RM3. As far as the average number of alleles per locus is concern, to some extent the same range of results were found by Ramadan et al. (2015), Nachimuthu et al. (2015) and Ngangkham et al. (2019).

Polymorphic information content (PIC) is the comparative measure of how much a marker is informative to distinguish between different populations and it depends upon the number of alleles detected by this marker and their relative frequency; thus, it provides an estimate of the discriminating power of the marker (Nagy et al., 2012). PIC values for the microsatellite markers used in this study varied from 0.00 to 0.743 with an average of 0.44. Markers with PIC values of 0.5 or higher are more useful in distinguishing the polymorphism rate of a marker at a specific locus (DeWoody et al. 1995), however, the mean polymorphism rate $(0.44)$ is found to be moderate in this study which are in agreement with the studies of Becerra (2015) and Adak (2020). The most appropriate explanation to this might be the similar origin, ecotype and speciation 


\section{Marker based genetic variability analysis of rice (Oryza sativa L.) landraces for drought tolerance}

of the land races. The highest PIC values were observed for RM252 (0.743) followed by RM505 (0.7), RM285 (0.648), RM254 (0.630), RM175 (0.613). In the present study according to the classification of Botstein et al. (1980), there was 14 numbers (35\% of total numbers of markers) of highly informative markers with PIC value range $>0.50$. On the other hand, 21 numbers of SSR markers with a PIC value $(0.50<$ PIC < $0.25)$ and 5 numbers of SSR markers with PIC value $(<0.25)$ proved themselves as moderately informative and slightly informative markers respectively.

Details of the microsatellite primers used in present study along with their allele size (bp), allele diversity, gene diversity and polymorphism information content (PIC) is depicted in Table (5). A significant correlation between PIC value and the total number of repeat motifs counts of di-nucleotide sequence per microsatellite marker was also revealed in the study. Loci amplifying di-nucleotide repeat motifs were found to be more polymorphic than those with tri-nucleotide and tetra-nucleotide repeat motifs. Most of the high values of PIC $(>0.5)$ were found to be associated with high numbers of repeated values of (GA) or (CT) dinucleotide sequences. These results, suggest that the total repeat count of SSR or microsatellite loci is associated with the high PIC value of particular marker. The larger the repeat numbers in the microsatellite DNA, the larger the number of identified alleles and PIC values. These results were consistent with those reported by Ramadan et al. (2015) and Melaku et al. (2018). Among the set of 40 numbers microsatellite primers tested in the study, RM 252 (PIC value of 0.743) with [(CT)19] repeat motifs, RM 505 (PIC value of 0.7$)$ with [(CT)12] repeat motif and RM285 (PIC value of 0.648) with [(GA)12] repeat motifs came out to be more informative as they could reveal more polymorphism in comparison to other primers. PCR amplified fragments produced by the some of the polymorphic markers in the current study are given in Figure 1(a,b) to Figure 3(a, b).

\section{Comparative analysis of molecular and morphological study:}

For carrying out a reproducible comparative analysis of the rice landraces under both morphological and molecular level, efforts have been rendered to analyse maximum possible numbers of small subgroups. In general, the dendrogram generated against the microsatellite based binary data produced more numbers of sub clusters (11 numbers) in comparison to morphological traits based dendrogram with 6 numbers of clusters (Fig. 4 and Fig. 5). Often, it has been observed, landraces, clustered in a single subgroup under morphological analysis are categorised into different subgroups under molecular analysis which signifies the better discrimination capacity of the microsatellite based molecular analysis over the morphology-based diversity analysis, however some similarities in clustering pattern was also been observed between those analyses. Four numbers of aromatic rice landraces viz. Kalikhasa, KhasaKasam, Kala Jira and American Ration clustered in a same group of $B_{1} B$ under morphological analysis, while under molecular analysis, only Kalikhasa and Khasakasam grouped together in a single cluster. Morphologically, there were ample similarities between the landraces of Kalikhasa and Kala Jira to raise the doubt on uniqueness of the landraces but better discrimination capacity of SSR based molecular analysis proved themselves as separate landraces under the study. The 'Fazu' group of landraces [FazuVom and Fazu Sen] grouped themselves in a single cluster under both morphological and molecular analysis, which probably signifies the same origin of the said land races.

Based on the mean value analysis of different significant yield governing 
Sujan Acharjee et al.

morphological traits and molecular analysis, a confirmatory analysis was also carried out to find out the correlation between the mean performance of the landraces and their clustering pattern under molecular analysis (Table 6). It has been revealed that the landraces viz. Kaporok, Releng, Tarkol, TarikolKolte, with more than $20 \mathrm{~cm}$ of total root length and Root: Culm ratio value of $>0.3$ clustered themselves into a single sub group of $B_{1}$. Yang Dhan and Badaya with moderate to high leaf drying index clustered themselves into a specific sub group of $\mathrm{C}_{2} \mathrm{~A}$. Kaporok, Releng, Tarkol,Beti are found to be clustered in a single sub group of $\mathrm{B}_{1}$ with low to moderate leaf rolling capacity.

But in this study, the main concern was to trace out the high yielder landraces and other yield contributing traits under moisture stress and as far as the grain yield under moisture stress condition is considered the landraces namely ChikanswariKabar, Garo Malati, Galong, Turkey, Tarkol, Dhalabalam, Maimi Hungar were found to be better performer under the study, out of which, ChikanswariKabar, the tallest and the highest yielder landrace settled itself in a single sub group of $\mathrm{C}_{2} \mathrm{C}$ under molecular analysis. Significantly, under morphological evaluation also, the said landrace grouped itself with only one high yielder landrace viz. Garo Malati. This certainly signifies the uniqueness and diverse nature of Chikanswarikabar from rest of the landraces and thus, can justifiably be utilised as a promising parent with expected heterosis effect in drought stress breeding programme. Interestingly, some landraces viz. Chikanswarikabar, Maimi Hungar, Garomalati, Kaporok and Tarkol which found to be better yielder under drought stress also showed comparatively deeper root length and higher root : culm ratio. This phenomenon probably justifies the better moisture utilising capacity of the landraces with improved root traits which ultimately resulted in better yield. Thus, those landraces also create a scope for further utilisation of those in future drought stress breeding programme.

It has also been found that, the landraces which shows slight to no leaf tip drying characters and slight to moderate leaf rolling capacity under moisture stress are basically good yielder. Along with, under this study, land races with intermediate to tall plant height also performed better as far as the grain yield is concern. Thus, along with grain yield, the better root attributes, leaf tip drying index (LDI), leaf rolling index (LRI) and plant height $(\mathrm{PH})$ may also be considered for selection of promising landraces under drought stress. No specific clustering patterns of landraces against the rest of the morphological traits numbers of productive tillers per plant, numbers of filled grains per plant etc were found. This signifies the polygenic nature of the quantitative characters and influence of environment on them.

Overall, the results obtained would help in the identification and differentiation of various cultivars being cultivated in this region to broaden the landrace base in the future of rice breeding programs. In addition, it will help in identifying efficient strategies for the sustainable management of the genetic resources of rice crops in the particular rain fed ecosystem of North East India

ABBREVIATIONS

(CT) - Cytosine and Thymine base, (GA) Guanine and Adenine base, dNTPsDeoxyribonucleotide triphosphate, RM: Rice Marker, TAE: Tris- Acetate, (Ethylenediaminetetraacetic Acid), Taq: Thermus aquaticus

Acknowledgement

Authors articulate honest gratitude to the Indian Council of Agriculture Research (ICAR) Complex for North Eastern Hilly Region, Tripura Centre for extending necessary support for conduction of the study in partial fulfilment of Ph.D. programme of $1^{\text {st }}$ author. 
Table 1: List of the 60 numbers of rice land races collected under the study and their collection sites

\begin{tabular}{|c|c|c|c|c|c|c|c|c|c|}
\hline$\dot{\bar{s}}$ & 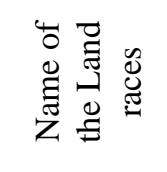 & $\begin{array}{l}\stackrel{0}{0} \\
\frac{0}{0} \\
\frac{0}{0}\end{array}$ & 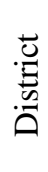 & 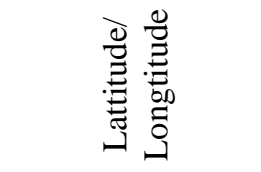 & $\dot{\sim}$ & 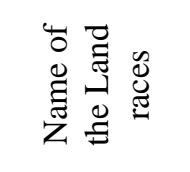 & 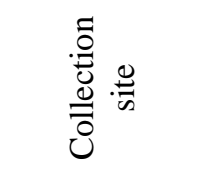 & 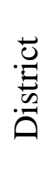 & 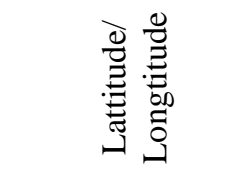 \\
\hline 1 & Kaporok & $\begin{array}{l}\text { Thamsarai } \\
\text { para }\end{array}$ & $\mathrm{N}$ & $\begin{array}{l}24^{\circ} 14^{\prime} \mathrm{N} \\
/ 92^{\circ} 17^{\prime} \mathrm{E}\end{array}$ & 31 & Darka Sona & $\begin{array}{l}\text { Thamsarai } \\
\text { para }\end{array}$ & $\mathrm{N}$ & $\begin{array}{l}24^{\circ} 14^{\prime} \mathrm{N} / \\
92^{\circ} 17^{\prime} \mathrm{E}\end{array}$ \\
\hline 2 & Releng & $\begin{array}{l}\text { Boithangbar } \\
\text { i }\end{array}$ & $\mathrm{N}$ & $24^{\circ} 19^{\prime} \mathrm{N} / 92^{\circ} 8^{\prime} \mathrm{E}$ & 32 & $\begin{array}{l}\text { Tarikol } \\
\text { Kolte }\end{array}$ & $\begin{array}{l}\text { Boithangbar } \\
\text { i }\end{array}$ & $\mathrm{N}$ & $\begin{array}{l}24^{\circ} 20^{\prime} \mathrm{N} \\
192^{\circ} 12^{\prime} \mathrm{E}\end{array}$ \\
\hline 3 & Beti & $\begin{array}{l}\text { Boithangbar } \\
\text { i }\end{array}$ & $\mathrm{N}$ & $24^{\circ} 19^{\prime} \mathrm{N} / 92^{\circ} 8^{\prime} \mathrm{E}$ & 33 & SadaBiroin & Ganganagar & $\mathrm{N}$ & $\begin{array}{l}24^{\circ} 20^{\prime} \mathrm{N} \\
/ 92^{\circ} 12^{\prime} \mathrm{E}\end{array}$ \\
\hline 4 & $\begin{array}{l}\text { MaimiUzr } \\
\text { a }\end{array}$ & $\begin{array}{l}\text { PurbaJalaiba } \\
\text { ri }\end{array}$ & $\mathrm{G}$ & $\begin{array}{l}23^{\circ} 30^{\prime} \mathrm{N} \\
/ 91^{\circ} 30^{\prime} \mathrm{E}\end{array}$ & 34 & $\begin{array}{l}\text { MaimiTauk } \\
\text { ha }\end{array}$ & $\begin{array}{l}\text { PurbaJalaiba } \\
\text { ri }\end{array}$ & G & $\begin{array}{l}23^{\circ} 12^{\prime} \mathrm{N} \\
/ 91^{\circ} 36^{\prime} \mathrm{E}\end{array}$ \\
\hline 5 & Kalikhasa & Ganganagar & $\mathrm{N}$ & $\begin{array}{l}24^{\circ} 20^{\prime} \mathrm{N} \\
/ 92^{\circ} 12^{\prime} \mathrm{E}\end{array}$ & 35 & Saluma & $\begin{array}{l}\text { PurbaJalaiba } \\
\text { ri }\end{array}$ & G & $\begin{array}{l}23^{\circ} 12^{\prime} \mathrm{N} \\
/ 91^{\circ} 36^{\prime} \mathrm{E}\end{array}$ \\
\hline 6 & Chinal & $\begin{array}{l}\text { Uttar Gakul } \\
\text { Nagar }\end{array}$ & W & $\begin{array}{l}23^{\circ} 42^{\prime} \mathrm{N} \\
/ 91^{\circ} 15^{\prime} \mathrm{E}\end{array}$ & 36 & Tarkol & Noagang & $\mathrm{N}$ & $\begin{array}{l}24^{\circ} 19^{\prime} \mathrm{N} / \\
92^{\circ} 13^{\prime} \mathrm{E}\end{array}$ \\
\hline 7 & American & Rajnagar & $\mathrm{N}$ & $24^{\circ} 19^{\prime} \mathrm{N} / 92^{\circ} 8^{\prime} \mathrm{E}$ & 37 & Madoop & Dalapati & $\mathrm{D}$ & $\begin{array}{l}23^{\circ} 37^{\prime} \mathrm{N} / \\
91^{\circ} 51^{\prime} \mathrm{E}\end{array}$ \\
\hline 8 & $\begin{array}{l}\text { KhasaKas } \\
\text { am }\end{array}$ & Kairai & W & $\begin{array}{l}23^{\circ} 51^{\prime} \mathrm{N} \\
/ 91^{\circ} 28^{\prime} \mathrm{E}\end{array}$ & 38 & Waibang & $\begin{array}{l}\text { Bilaiham } \\
\text { para }\end{array}$ & W & $\begin{array}{l}23^{\circ} 52^{\prime} \mathrm{N} / \\
91^{\circ} 41^{\prime} \mathrm{E}\end{array}$ \\
\hline 9 & Biroin & Rajnagar & $\mathrm{N}$ & $\begin{array}{l}24^{\circ} 20^{\prime} \mathrm{N} \\
/ 92^{\circ} 12^{\prime} \mathrm{E}\end{array}$ & 39 & Jhum Bini & Garjeecherra & G & $\begin{array}{l}23^{\circ} 26^{\prime} \mathrm{N} \\
/ 91^{\circ} 30^{\prime} \mathrm{E}\end{array}$ \\
\hline 10 & Galong & $\begin{array}{l}\text { Boithangbar } \\
\text { i }\end{array}$ & $\mathrm{N}$ & $\begin{array}{l}24^{\circ} 20^{\prime} \mathrm{N} \\
/ 92^{\circ} 12^{\prime} \mathrm{E}\end{array}$ & 40 & $\begin{array}{l}\text { Fazu Sen } \\
\text { (White) }\end{array}$ & $\begin{array}{l}\text { Athramura } \\
\text { range }\end{array}$ & $\mathrm{K}$ & $\begin{array}{l}23^{\circ} 51 ' \mathrm{~N} / \\
91^{\circ} 44^{\prime} \mathrm{E}\end{array}$ \\
\hline 11 & FazuVom & $\begin{array}{l}\text { Boithangbar } \\
\text { i }\end{array}$ & $\mathrm{N}$ & $\begin{array}{l}24^{\circ} 20^{\prime} \mathrm{N} / \\
92^{\circ} 12^{\prime} \mathrm{E}\end{array}$ & 41 & Bongbu & Noagang & $\mathrm{N}$ & $\begin{array}{l}24^{\circ} 19^{\prime} \mathrm{N} \\
/ 92^{\circ} 13^{\prime} \mathrm{E}\end{array}$ \\
\hline 12 & $\begin{array}{l}\text { Garo } \\
\text { Malati }\end{array}$ & $\begin{array}{l}\text { Uttar Gakul } \\
\text { Nagar }\end{array}$ & W & $\begin{array}{l}23^{\circ} 42^{\prime} \mathrm{N} / \\
91^{\circ} 15^{\prime} \mathrm{E}\end{array}$ & 42 & Sadok & Noagang & $\mathrm{N}$ & $\begin{array}{l}24^{\circ} 19^{\prime} \mathrm{N} \\
/ 92^{\circ} 13^{\prime} \mathrm{E}\end{array}$ \\
\hline 13 & $\begin{array}{l}\text { Maimi } \\
\text { Usha }\end{array}$ & $\begin{array}{l}\text { PurbaJalaiba } \\
\text { ri }\end{array}$ & $\mathrm{G}$ & $\begin{array}{l}23^{\circ} 12^{\prime} \mathrm{N} \\
191^{\circ} 36^{\prime} \mathrm{E}\end{array}$ & 43 & Kala Jira & Kameswar & $\mathrm{N}$ & $\begin{array}{l}24^{\circ} 22^{\prime} \mathrm{N} / \\
92^{\circ} 11^{\prime} \mathrm{E}\end{array}$ \\
\hline 14 & $\begin{array}{l}\text { Maimi } \\
\text { Red }\end{array}$ & $\begin{array}{l}\text { Thamsarai } \\
\text { para }\end{array}$ & $\mathrm{N}$ & $\begin{array}{l}24^{\circ} 14^{\prime} \mathrm{N} \\
192^{\circ} 17^{\prime} \mathrm{E}\end{array}$ & 44 & Gaigash & $\begin{array}{l}\text { Murasingh } \\
\text { Para }\end{array}$ & G & $23^{\circ} 42^{\prime} \mathrm{N} / 91^{\circ} 43 \mathrm{E}$ \\
\hline 15 & Suri & $\begin{array}{l}\text { Aswinirojap } \\
\text { ara }\end{array}$ & $\mathrm{D}$ & $\begin{array}{l}23^{\circ} 34^{\prime} \mathrm{N} / \\
91^{\circ} 54^{\prime} \mathrm{E}\end{array}$ & 45 & Vanbang & Killa & $\mathrm{S}$ & $\begin{array}{l}23^{\circ} 36^{\prime} \mathrm{N} \\
/ 91^{\circ} 31^{\prime} \mathrm{E}\end{array}$ \\
\hline 16 & Lebuka & $\begin{array}{l}\text { Thamsarai } \\
\text { para }\end{array}$ & $\mathrm{N}$ & $\begin{array}{l}24^{\circ} 14^{\prime} \mathrm{N} \\
/ 92^{\circ} 17^{\prime} \mathrm{E}\end{array}$ & 46 & Makajaria & Rajnagar & $\mathrm{N}$ & $24^{\circ} 19^{\prime} \mathrm{N} / 92^{\circ} 7^{\prime} \mathrm{E}$ \\
\hline 17 & Aaduma & $\begin{array}{l}\text { Aswinirojap } \\
\text { ara }\end{array}$ & $\mathrm{D}$ & $\begin{array}{l}23^{\circ} 34^{\prime} \mathrm{N} \\
191^{\circ} 54^{\prime} \mathrm{E}\end{array}$ & 47 & Jilong & $\begin{array}{l}\text { Bilaiham } \\
\text { para }\end{array}$ & $\mathrm{W}$ & $\begin{array}{l}23^{\circ} 42^{\prime} \mathrm{N} / \\
91^{\circ} 15^{\prime} \mathrm{E}\end{array}$ \\
\hline 18 & Fazu Sen & $\begin{array}{l}\text { Boithangbar } \\
\text { i }\end{array}$ & $\mathrm{N}$ & $\begin{array}{l}24^{\circ} 20^{\prime} \mathrm{N} \\
/ 92^{\circ} 12^{\prime} \mathrm{E}\end{array}$ & 48 & $\begin{array}{l}\text { American } \\
\text { Ration }\end{array}$ & $\begin{array}{l}\text { Thamsarai } \\
\text { para }\end{array}$ & $\mathrm{N}$ & $\begin{array}{l}24^{\circ} 14^{\prime} \mathrm{N} / \\
92^{\circ} 17^{\prime} \mathrm{E}\end{array}$ \\
\hline 19 & FazuNgoi & $\begin{array}{l}\text { Boithangbar } \\
\text { i }\end{array}$ & $\mathrm{N}$ & $\begin{array}{l}24^{\circ} 20^{\prime} \mathrm{N} / \\
92^{\circ} 12^{\prime} \mathrm{E}\end{array}$ & 49 & Kala Dhan & $\begin{array}{l}\text { Bilaiham } \\
\text { para }\end{array}$ & W & $\begin{array}{l}23^{\circ} 42^{\prime} \mathrm{N} / \\
91^{\circ} 15^{\prime} \mathrm{E}\end{array}$ \\
\hline 20 & BetiKalai & $\begin{array}{l}\text { Brahmacharr } \\
\text { a }\end{array}$ & $\mathrm{K}$ & $\begin{array}{l}23^{\circ} 48^{\prime} \mathrm{N} \\
/ 91^{\circ} 39^{\prime} \mathrm{E}\end{array}$ & 50 & Turkey & Dalapati & $\mathrm{D}$ & $\begin{array}{l}23^{\circ} 37^{\prime} \mathrm{N} / \\
91^{\circ} 51^{\prime} \mathrm{E}\end{array}$ \\
\hline 21 & $\begin{array}{l}\text { Saanki ka } \\
\text { Phool }\end{array}$ & Dalapati & $\mathrm{D}$ & $\begin{array}{l}23^{\circ} 37^{\prime} \mathrm{N} / \\
91^{\circ} 51^{\prime} \mathrm{E}\end{array}$ & 51 & $\begin{array}{l}\text { SaankiKach } \\
\text { ak }\end{array}$ & Dalapati & $\mathrm{D}$ & $\begin{array}{l}23^{\circ} 37^{\prime} \mathrm{N} / \\
91^{\circ} 51^{\prime} \mathrm{E}\end{array}$ \\
\hline 22 & Bihar & $\begin{array}{l}\text { Brahmacharr } \\
\text { a }\end{array}$ & $\mathrm{K}$ & $\begin{array}{l}23^{\circ} 48^{\prime} \mathrm{N} \\
/ 91^{\circ} 39^{\prime} \mathrm{E}\end{array}$ & 52 & $\begin{array}{l}\text { MaimiUkhl } \\
\text { ao }\end{array}$ & $\begin{array}{l}\text { PurbaJalaiba } \\
\text { ri }\end{array}$ & G & $\begin{array}{l}23^{\circ} 12^{\prime} \mathrm{N} \\
191^{\circ} 36^{\prime} \mathrm{E}\end{array}$ \\
\hline 23 & $\begin{array}{l}\text { Chikansw } \\
\text { ariKabar }\end{array}$ & Harbang & W & $\begin{array}{l}23^{\circ} 54^{\prime} \mathrm{N} \\
191^{\circ} 31^{\prime} \mathrm{E}\end{array}$ & 53 & Maiwasha & $\begin{array}{l}\text { Old Dalapati } \\
\text { Para }\end{array}$ & $\mathrm{D}$ & $\begin{array}{l}23^{\circ} 37^{\prime} \mathrm{N} / \\
91^{\circ} 51^{\prime} \mathrm{E}\end{array}$ \\
\hline
\end{tabular}


Sujan Acharjee et al.

\begin{tabular}{|c|c|c|c|c|c|c|c|c|c|}
\hline 24 & $\begin{array}{l}\text { Bangbu } \\
\text { Jhum }\end{array}$ & $\begin{array}{l}\text { Nunnacharra } \\
\text { Range }\end{array}$ & $\mathrm{K}$ & $\begin{array}{l}23^{\circ} 46^{\prime} \mathrm{N} / \\
91^{\circ} 44^{\prime} \mathrm{E}\end{array}$ & 54 & $\begin{array}{l}\text { MaimiWato } \\
\text { lok }\end{array}$ & Dalapati & $\mathrm{D}$ & $\begin{array}{l}23^{\circ} 37^{\prime} \mathrm{N} / \\
91^{\circ} 51^{\prime} \mathrm{E}\end{array}$ \\
\hline 25 & Lal Biroin & Kameswar & $\mathrm{N}$ & $\begin{array}{l}24^{\circ} 22^{\prime} \mathrm{N} \\
192^{\circ} 11^{\prime} \mathrm{E}\end{array}$ & 55 & $\begin{array}{l}\text { Santinmaw } \\
\text { Wakhum }\end{array}$ & Killa & $\mathrm{S}$ & $\begin{array}{l}23^{\circ} 36^{\prime} \mathrm{N} \\
191^{\circ} 31^{\prime} \mathrm{E}\end{array}$ \\
\hline 26 & $\begin{array}{l}\text { DhalaBala } \\
\mathrm{m}\end{array}$ & Rajnagar & $\mathrm{N}$ & $24^{\circ} 19^{\prime} \mathrm{N} / 92^{\circ} 7^{\prime} \mathrm{E}$ & 56 & Yang Dhan & Killa & $\mathrm{S}$ & $\begin{array}{l}23^{\circ} 36^{\prime} \mathrm{N} \\
191^{\circ} 31^{\prime} \mathrm{E}\end{array}$ \\
\hline 27 & Goria & $\begin{array}{l}\text { Char Garia } \\
\text { Para }\end{array}$ & $\mathrm{W}$ & $\begin{array}{l}23^{\circ} 54^{\prime} \mathrm{N} / \\
91^{\circ} 31^{\prime} \mathrm{E}\end{array}$ & 57 & Badaya & Killa & $\mathrm{S}$ & $\begin{array}{l}23^{\circ} 366^{\prime} \mathrm{N} / \\
91^{\circ} 31^{\prime} \mathrm{E}\end{array}$ \\
\hline 28 & Bahadur & $\begin{array}{l}\text { South Ganga } \\
\text { Nagar }\end{array}$ & $\mathrm{N}$ & $\begin{array}{l}24^{\circ} 20^{\prime} \mathrm{N} \\
192^{\circ} 12^{\prime} \mathrm{E}\end{array}$ & 58 & Kanchali & Killa & $\mathrm{S}$ & $\begin{array}{l}23^{\circ} 36^{\prime} \mathrm{N} \\
191^{\circ} 31^{\prime} \mathrm{E}\end{array}$ \\
\hline 29 & $\begin{array}{l}\text { Maimiwat } \\
\text { oklokMan } \\
\text { doori }\end{array}$ & $\begin{array}{l}\text { Brahmacharr } \\
\text { a }\end{array}$ & $\mathrm{K}$ & $\begin{array}{l}23^{\circ} 48^{\prime} \mathrm{N} / \\
91^{\circ} 39^{\prime} \mathrm{E}\end{array}$ & 59 & $\begin{array}{l}\text { Australian } \\
\text { Biroin }\end{array}$ & Kameswar & $\mathrm{N}$ & $\begin{array}{l}24^{\circ} 22^{\prime} \mathrm{N} \\
/ 92^{\circ} 11^{\prime} \mathrm{E}\end{array}$ \\
\hline 30 & $\begin{array}{l}\text { MaimiHu } \\
\text { ngar }\end{array}$ & $\begin{array}{l}\text { Twisarangch } \\
\text { ak para }\end{array}$ & W & $\begin{array}{l}23^{\circ} 54^{\prime} \mathrm{N} / \\
91^{\circ} 31^{\prime} \mathrm{E}\end{array}$ & 60 & $\begin{array}{l}\text { Assam } \\
\text { Paisom }\end{array}$ & Rajnagar & $\mathrm{N}$ & $24^{\circ} 19^{\prime} \mathrm{N} / 92^{\circ} 7^{\prime} \mathrm{E}$ \\
\hline
\end{tabular}

D-Dhalai, G- Gomati,K-Khowai,N- North Tripura, S-South Tripura,W-West Tripura Source: Acharjee $e t$ al.(2019)

Table 2: Pulled mean values of 15 (Fifteen) numbers of morphological characters of 60 (sixty) land races under drought stress condition

\begin{tabular}{|c|c|c|c|c|c|c|c|c|c|c|c|c|c|c|c|c|}
\hline $\begin{array}{l}\dot{2} \\
\dot{z} \\
\dot{\omega}\end{array}$ & $\frac{2}{0}$ & $\stackrel{0}{3}$ & $\vec{a}$ & $\overline{3}$ & $\begin{array}{l}5 \\
\text { 至 } \\
\text { 足 }\end{array}$ & $\begin{array}{l}\stackrel{\cong}{E} \\
\stackrel{2}{0}\end{array}$ & $\begin{array}{l}z \\
z \\
z\end{array}$ & $\begin{array}{l}\frac{2}{m} \\
\frac{\alpha}{2}\end{array}$ & $\frac{z}{2}$ & 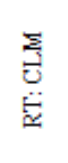 & 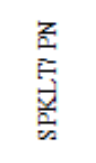 & 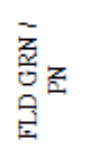 & 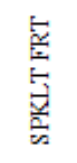 & $\begin{array}{l}5 \\
9 \\
8 \\
8\end{array}$ & $\Xi$ & $\begin{array}{l}\stackrel{5}{\leftrightarrows} \\
\stackrel{5}{0}\end{array}$ \\
\hline 1 & Kaporok & 5.50 & 2.00 & 0.25 & 94.67 & 4.33 & 23.33 & 7.50 & 22.15 & 0.31 & 103.50 & 90.42 & 87.63 & 2.22 & 26.10 & 9.31 \\
\hline 2 & Releng & $7.00^{\circ}$ & 1.00 & $0.00^{\circ}$ & 87.08 & 3.75 & 23.75 & $7.91^{-}$ & 21.23 & 0.34 & 103.17 & 72.59 & 70.35 & $2.60^{\circ}$ & 26.51 & 7.53 \\
\hline 3 & Beti & $5.00^{\circ}$ & 3.00 & $0.00^{\circ}$ & 85.33 & 3.92 & 20.42 & 6.84 & $19.26^{-}$ & $0.30^{\circ}$ & $91.00^{-}$ & 80.75 & 88.75 & 2.43 & 24.90 & 7.87 \\
\hline 4 & MaimiUza & $7.00^{\circ}$ & 0.00 & $1.25^{\circ}$ & 102.08 & $3.25^{-}$ & 23.75 & 8.08 & 15.01 & $0.19^{-}$ & $84.08^{-}$ & $68.50^{-}$ & 81.54 & 2.22 & 24.06 & 5.51 \\
\hline 5 & Kalikhasa & $7.50^{\circ}$ & 2.00 & $2.00^{\circ}$ & 84.92 & 3.92 & 17.08 & 5.67 & 10.58 & 0.16 & 80.17 & 69.33 & 86.49 & 1.10 & 13.63 & 3.23 \\
\hline 6 & "Chinal" & 6.50 & 0.00 & 3.50 & $80^{\circ} .50^{\circ}$ & 3.58 & 24.42 & 6.33 & 11.51 & 0.21 & 65.08 & 54.75 & 84.07 & 2.19 & $16.42^{-}$ & 4.65 \\
\hline 7 & American & 8.00 & 0.00 & 0.00 & 85.25 & 2.83 & 24.92 & 6.58 & 13.42 & 0.22 & 114.34 & 88.08 & 77.11 & 2.33 & 25.84 & 6.31 \\
\hline 8 & Khäarasam & $8.00^{-}$ & $0.50^{-}$ & $0.50^{-}$ & 75.75 & 3.33 & 16.83 & $5.58^{-}$ & 10.32 & 0.19 & $80.177^{-1}$ & 66.08 & 82.18 & 1.26 & 13.88 & 2.97 \\
\hline 9 & Biroin & $6.50^{-}$ & 0.00 & $0.00^{\circ}$ & 85.25 & 3.92 & 25.25 & 7.75 & 21.08 & 0.35 & 90.67 & 78.67 & 86.74 & 2.35 & 27.49 & 7.48 \\
\hline 10 & Galong & $6.50^{-}$ & 3.00 & 0.25 & 100.91 & 3.42 & 26.17 & 7.92 & 21.62 & 0.29 & 164.33 & 147.42 & 89.51 & 2.69 & 39.07 & 14.08 \\
\hline $\mathrm{Tl}^{-}$ & FaziVom & 7.50 & 2.00 & 1.00 & 69.75 & 3.42 & 23.75 & 7.17 & 10.76 & 0.24 & 64.42 & 51.67 & 80.19 & 2.39 & $21.60^{\circ}$ & 4.58 \\
\hline 12 & "Garo Malati" & 5.00 & 1.00 & $0.00^{\circ}$ & 124.16 & 4.42 & 29.17 & $9.33^{-1}$ & 26.53 & 0.28 & 162.83 & 147.75 & $90.84^{-}$ & 2.75 & 34.85 & 18.63 \\
\hline $13^{-}$ & Maim Usha & 8.00 & 1.00 & 1.50 & 61.25 & $3.50^{\circ}$ & 23.25 & 6.92 & 8.69 & 0.23 & 94.75 & 80.75 & 85.24 & 2.19 & $28.88^{-}$ & $6.66^{-}$ \\
\hline 14 & Mămi Red & $7.50^{\circ}$ & $1.00^{\circ}$ & $0.50^{\circ}$ & $94.50^{\circ}$ & $3.50^{\circ}$ & 23.16 & $6.42^{-}$ & 18.58 & 0.26 & $77.83^{-}$ & 65.02 & 83.54 & $2.89^{-}$ & 23.72 & 6.65 \\
\hline 15 & Suri & 8.50 & 0.00 & $1.00^{-}$ & 73.00 & 3.58 & 20.50 & $6.00^{-}$ & 10.62 & $0.20^{\circ}$ & $76.67^{\circ}$ & 63.09 & 82.23 & $2.10^{\circ}$ & 22.12 & 5.39 \\
\hline 16 & Lebulka & $7.00^{\circ}$ & 0.25 & $0.00^{\circ}$ & 83.33 & $3.50^{\circ}$ & 26.08 & 7.25 & 8.24 & $0.15^{-}$ & 85.08 & 67.83 & 79.72 & 2.05 & 20.60 & 5.58 \\
\hline 17 & Asduma & $7.00^{\circ}$ & 0.00 & $2.00^{\circ}$ & 79.33 & $4.25^{-}$ & 22.58 & 6.67 & $15.78^{-}$ & 0.28 & 78.91 & $65.91^{-}$ & 82.28 & 1.67 & 19.07 & 5.00 \\
\hline 18 & Fazid Sen & $8.00^{\circ}$ & 1.00 & $1.00^{\circ}$ & 83.92 & 3.08 & 22.34 & 5.92 & 14.17 & 0.23 & 65.25 & 49.67 & 76.35 & 2.73 & 19.45 & 4.91 \\
\hline 19 & FazuNG & 9.00 & 2.50 & 2.00 & 77.25 & 2.83 & 20.08 & $7.92^{-}$ & 14.96 & 0.27 & 63.33 & 54.09 & 85.06 & 2.35 & 19.62 & 4.29 \\
\hline $20^{-}$ & BetiRalai & 6.00 & 0.50 & 0.50 & 91.08 & 3.67 & 23.75 & 6.33 & 16.03 & 0.24 & 95.92 & 83.34 & 87.02 & 2.75 & 28.95 & 8.88 \\
\hline $21^{-}$ & $\begin{array}{l}\text { Saanlik ka } \\
\text { Phool }\end{array}$ & 8.00 & 0.00 & 0.00 & 79.83 & 3.58 & 22.42 & 6.33 & 8.40 & 0.15 & 62.00 & 49.75 & 79.95 & 2.09 & 16.07 & 4.19 \\
\hline 22 & Bihar & $7.00^{\circ}$ & 1.00 & $0.50^{\circ}$ & 86.58 & 3.58 & 20.17 & 5.25 & 12.12 & 0.18 & 69.92 & $55.92^{-}$ & 80.40 & 1.78 & 16.17 & 4.56 \\
\hline $23^{-}$ & $\begin{array}{l}\text { Chikanswark } \\
\text { abar }\end{array}$ & 5.50 & 0.50 & 0.50 & 125.42 & 4.50 & 29.50 & 10.25 & 24.76 & 0.26 & 195.33 & 178.58 & 91.43 & 2.52 & 40.86 & 21.66 \\
\hline 24 & Bangbu Jhum & $6.50^{-}$ & 0.00 & $1.00^{\circ}$ & 77.33 & 2.92 & 22.25 & $6.50^{-}$ & 12.82 & 0.23 & $57.50^{\circ}$ & 45.42 & -79.53 & 2.78 & 21.39 & 3.94 \\
\hline 25 & Lal Biroin & $7.00^{\circ}$ & 0.00 & $2.50^{\circ}$ & 78.75 & 3.17 & 17.67 & 7.42 & 11.71 & 0.19 & 80.25 & $66.50^{-}$ & 82.89 & 2.55 & 25.46 & 5.75 \\
\hline $26^{-}$ & DhälaBalam & $6.50^{\prime}$ & 0.50 & 0.25 & 83.42 & 4.08 & 22.58 & $7.75^{\prime}$ & $12.30^{\circ}$ & $0.20^{\circ}$ & 96.83 & $83.92^{-}$ & 86.64 & 2.77 & 30.49 & 10.00 \\
\hline $27^{\circ}$ & Goria & 7.00 & 0.00 & 0.50 & 94.92 & 3.41 & 19.67 & 5.33 & 9.73 & 0.13 & 68.67 & 56.92 & 83.03 & 2.44 & $22.30^{\circ}$ & 5.18 \\
\hline 28 & Bahadur & $9.00^{\circ}$ & 0.00 & $1.50^{\circ}$ & 95.00 & 4.00 & 25.16 & 6.42 & 14.08 & 0.20 & $65.67^{-1}$ & 51.00 & 77.72 & 1.82 & 12.80 & 3.76 \\
\hline 29 & $\begin{array}{l}\text { M.W.Mand } \\
\text { oori }\end{array}$ & 7.00 & 0.00 & 2.00 & 91.83 & 3.83 & 27.50 & 8.58 & 9.65 & 0.15 & 98.42 & 79.42 & 80.05 & 2.16 & 27.07 & 6.96 \\
\hline 30 & $\begin{array}{l}\text { Mami } \\
\text { Hungar }\end{array}$ & 5.50 & 0.50 & 0.50 & 98.42 & 3.92 & 28.92 & 8.67 & 23.13 & 0.33 & 03.33 & 1.50 & 8.49 & 2.99 & 3.76 & 1.04 \\
\hline
\end{tabular}

VGR- Seedling vegetative vigour, LRI- Leaf rolling index, LDI-Leaf tip drying index, PL HT - Plant Height (cm.), PR TLR-Numbers of Productive Tiller per plant, PN LN -Panicle length $(\mathrm{cm}$.), PRI BR-Numbers of Primary branch per panicle, RT LN-Root length $(\mathrm{cm}$.), RT:CLM-Root- Culm ratio, SPKLT/PN-Numbers of Spike lets per panicle, FLD GRN/ PN-Numbers of filled grain per panicle, SPKLT FRT-Spikelet fertility (\%),100 SD WT-100 Seed's weight (gm.), HI-Harvest Index (\%),GR Y /PLT-Grain yield per plant (gm.) 


\section{Marker based genetic variability analysis of rice (Oryza sativa L.) landraces for drought tolerance}

Table 2: cont. Pulled mean value of 15 (Fifteen) numbers of morphological characters of 60 (sixty) land races under drought stress condition

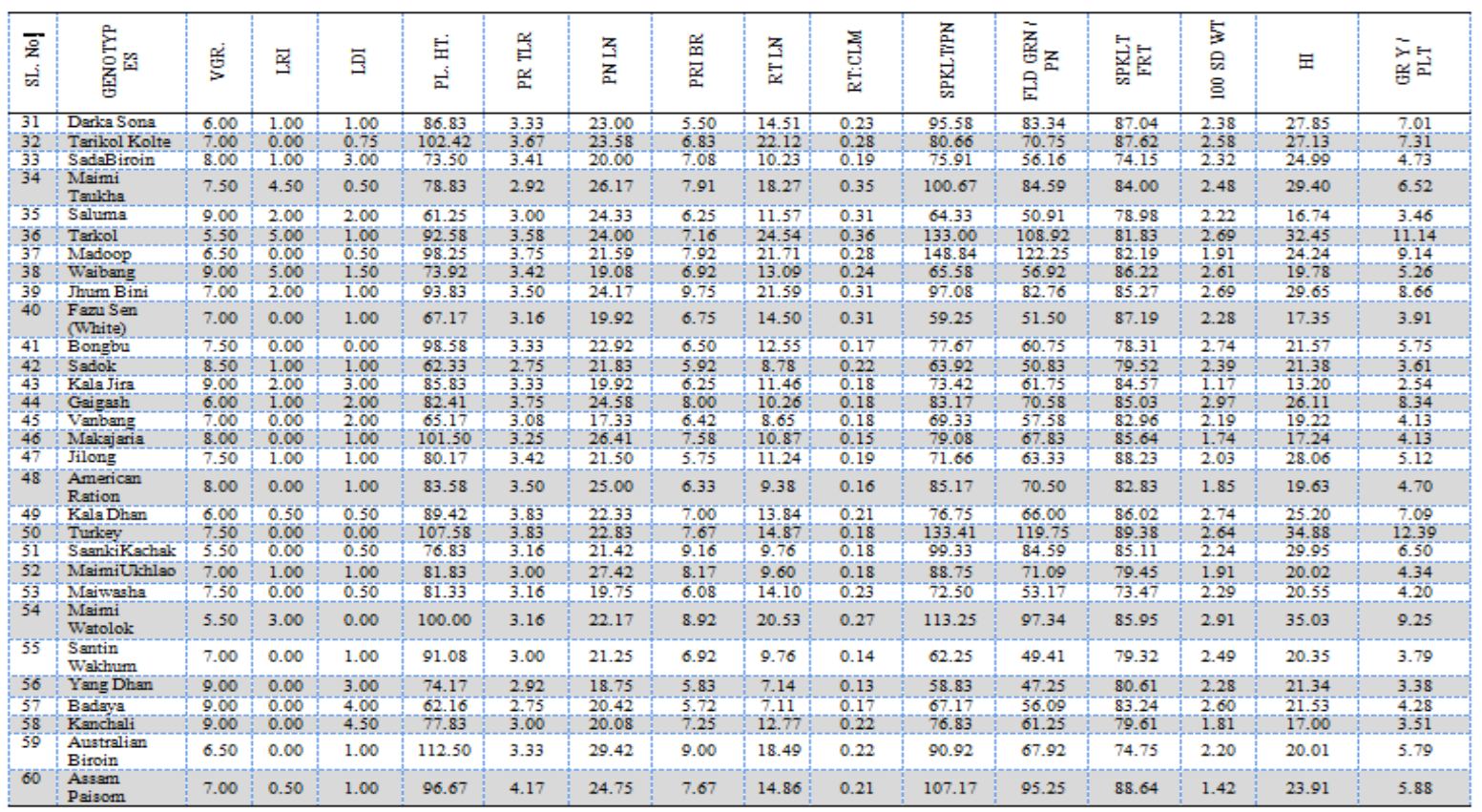

VGR-Seedling vegetative vigour, LRI- Leaf rolling index, LDI-Leaf tip drying index, PL HT - Plant Height (cm.), PR TLR-Numbers of Productive Tiller per plant, PN LN -Panicle length (cm.), PRI BR-Numbers of Primary branch per panicle, RT LN-Root length (cm.), RT:CLM-Root- Culm ratio, SPKLT/PN-Numbers of Spike lets per panicle, FLD GRN/ PN-Numbers of filled grain per panicle, SPKLT FRT-Spikelet fertility (\%), 100 SD WT-100 Seed's weight (gm.), HI-Harvest Index (\%),GR Y /PLT-Grain yield per plant (gm.)

Table 3: Estimates of Genetic parameters for 15 (Fifteen) numbers of Morpho-Physiological characters of 60 (sixty) land races under drought stress condition

\begin{tabular}{|c|c|c|c|c|c|c|c|c|c|}
\hline \multirow[b]{2}{*}{ Character } & \multicolumn{2}{|c|}{ Range } & \multirow{2}{*}{$\begin{array}{l}\text { Grand } \\
\text { Mean }\end{array}$} & \multirow{2}{*}{$\begin{array}{l}\text { Expected } \\
\text { mean next } \\
\text { generation }\end{array}$} & \multirow[b]{2}{*}{$\mathrm{CV}$} & \multirow[b]{2}{*}{ GCV } & \multirow[b]{2}{*}{ PCV } & \multirow[b]{2}{*}{$\mathrm{H}^{2}$} & \multirow[b]{2}{*}{$\begin{array}{c}\text { GA as } \% \text { of mean } \\
5 \%\end{array}$} \\
\hline & Min. & Max. & & & & & & & \\
\hline VGR. & 5.0 & 9.0 & 7.17 & 8.76 & 12.06 & 14.15 & 18.60 & 0.57 & 22.19 \\
\hline LRI & 0.00 & 5.00 & 0.89 & 3.28 & 50.81 & 137.95 & 147.01 & 0.88 & 266.66 \\
\hline LDI & 0.00 & 4.50 & 1.11 & 2.62 & 71.99 & 86.06 & 112.20 & 0.58 & 135.99 \\
\hline PL. HT. & 61.24 & 125.41 & 86.17 & 108.39 & 10.29 & 15.14 & 18.31 & 0.68 & 25.79 \\
\hline PR TLR & 2.74 & 4.5 & 3.47 & 3.95 & 12.44 & 10.51 & 16.29 & 0.41 & 13.98 \\
\hline PN LN & 16.83 & 29.49 & 22.87 & 27.51 & 9.45 & 12.40 & 15.59 & 0.63 & 20.31 \\
\hline PRI BR & 5.25 & 10.24 & 7.11 & 8.84 & 11.51 & 14.95 & 18.87 & 0.62 & 24.41 \\
\hline RT LN & 7.11 & 26.52 & 14.28 & 23.56 & 15.17 & 34.44 & 37.64 & 0.83 & 64.94 \\
\hline RT: CLM & 0.13 & 0.35 & 0.22 & 0.34 & 8.22 & 26.76 & 27.99 & 0.91 & 52.69 \\
\hline SPKLT/PN & 57.49 & 195.33 & 88.51 & 139.88 & 13.25 & 30.68 & 33.42 & 0.84 & 58.03 \\
\hline FLD GRN/ PN & 45.41 & 178.58 & 74.08 & 122.64 & 14.30 & 34.45 & 37.30 & 0.85 & 65.55 \\
\hline SPKLT FRT & 70.34 & 91.43 & 83.06 & 90.27 & 3.52 & 5.117 & 6.21 & 0.67 & 8.67 \\
\hline $100 \mathrm{SD} W \mathrm{WT}$ & 1.10 & 2.99 & 2.29 & 3.13 & 6.36 & 18.60 & 19.65 & 0.89 & 36.25 \\
\hline HI & 12.8 & 40.86 & 23.81 & 34.74 & 14.50 & 25.61 & 29.43 & 0.75 & 45.90 \\
\hline GR Y /PLT & 2.53 & 21.66 & 6.53 & 13.03 & 23.40 & 52.77 & 57.72 & 0.83 & 99.37 \\
\hline
\end{tabular}

Min-Minimum, Max- Maximum, CV-Coefficient of variance, GCV - genotypic coefficient of variations PCVphenotypic coefficient of variations, $\mathrm{H}^{2}-$ Broad sense heritability in percentage, $\mathrm{GA}-$ Genetic advance 
Sujan Acharjee et al.

Table 4: Grouping of landraces based on Mean value analysis of some significant morphological traits

\begin{tabular}{|c|c|}
\hline \multicolumn{2}{|c|}{ LEAF ROLLING AT VEGETATIVE STAGE } \\
\hline No sign of leaf rolling & $\begin{array}{l}\text { MaimiUzra, Chinal, American, Biroin, Aaduma, Suri, Saanki ka phool, } \\
\text { Bangbu Jhum, Lal Biroin, Goria, Bahadur, Mami WatoklokMandoori, } \\
\text { Bangbu, Vanbang, Makajaraia, Turkey, SaankiKachak, SaantinWakhom, } \\
\text { Yang dhan, Badaya, Kanchali, Australian Biroin }\end{array}$ \\
\hline Slight leaf rolling & $\begin{array}{l}\text { KhasaKasam, Lebuka, BetiKalai, ChikanswariKabar, DhalaBalam, } \\
\text { MaimiHungar, Kaladhan, Assam Paisom }\end{array}$ \\
\hline Moderate leaf rolling & $\begin{array}{l}\text { Tarkol, Waibang, MaimiWatoklok, Galong, Beti, Kaporok, Kalikhasa, Sadok, } \\
\text { FazuVom, Kalajira }\end{array}$ \\
\hline \multicolumn{2}{|c|}{ LEAF DRYING AT VEGETATIVE STAGE } \\
\hline No sign of leaf drying & $\begin{array}{l}\text { Releng, Beti, American, Biroin, Garomalati, Lebuka, Saanki ka phool, } \\
\text { Bangbu, MaimiWatoklok, Turkey }\end{array}$ \\
\hline Slight leaf drying & $\begin{array}{l}\text { Maimi Red, BetiKalai, Bihar, ChikanswariKabar, Goria, MaimiHungar, } \\
\text { MaimiTaukha, Madoop, Waibang, Kaladhan, Maiwasha }\end{array}$ \\
\hline $\begin{array}{l}\text { Moderate to high leaf } \\
\text { drying }\end{array}$ & Chinal, SadaBiroin, Kala Jira, Yang Dhan, Badaya, Kanchali \\
\hline \multicolumn{2}{|l|}{ ROOT LENGTH } \\
\hline $\begin{array}{l}\text { More than } 20 \mathrm{~cm} \text {. under } \\
\text { drought stress }\end{array}$ & $\begin{array}{l}\text { Kaporok, Releng, Garomalati, ChikanswariKabar, MaimiHungar, Tarikol } \\
\text { Kolte, Tarkol, Madoop, MaimiWatoklok }\end{array}$ \\
\hline \multicolumn{2}{|l|}{ PLANT HEIGHT } \\
\hline Tall (More than $125 \mathrm{~cm})$. & ChikanswariKabar \\
\hline $\begin{array}{l}\text { Intermediate } \\
(90-125) \mathrm{cm} .\end{array}$ & $\begin{array}{l}\text { Garo malati, Kaporok, MaimiUzra, Galong,BetiKalai,Goria, Bahdur, } \\
\text { M.W.Mandoori, MaimiHungar, Tarikolkolte, Tarkol, Madoop, Jhum Bini, } \\
\text { Bangbu, Makajaria, Turkey, MaimiWatoklok, SantinWakhum, Australian } \\
\text { Biroin, Assam Paisom }\end{array}$ \\
\hline $\begin{array}{c}\text { Semidwarf } \\
\text { (Less than } 90 \mathrm{~cm} .)\end{array}$ & Rest of the landraces \\
\hline \multicolumn{2}{|l|}{ GRAIN YIELD PER PLANT } \\
\hline More than $10 \mathrm{gm}$ & $\begin{array}{l}\text { ChikanswariKabar, Garomalati, Galong, Turkey, Tarkol } \\
\text {,DhalaBalam,MaimiHungar. }\end{array}$ \\
\hline \multicolumn{2}{|l|}{100 SEED WEIGHT } \\
\hline More than $2.5 \mathrm{gm}$. & $\begin{array}{l}\text { MaimiHungar, Gaigash, MaimiWatoklok, Maimi Red, Bangbu jhum, Releng, } \\
\text { Galong, Garo Malati, Fazu Sen, BetiKalai, ChikanswariKabar, Lal Biroin, } \\
\text { DhalaBalam, Tarikolkolte, Tarkol, Waibang, Jhum Bini, Bongbu, Kala dhan, } \\
\text { Turkey, Badya }\end{array}$ \\
\hline \multicolumn{2}{|l|}{ ROOT: CULM RATIO } \\
\hline More than 0.3 & $\begin{array}{l}\text { Tarkol, Releng, MaimiHungar, MaimiTaukha, Kaporok, Jhum Bini, Fazu Sen } \\
\text { (white) }\end{array}$ \\
\hline \multicolumn{2}{|c|}{ NUMBERS OF FILLED GRAIN PER PANICLE } \\
\hline \multicolumn{2}{|l|}{$\begin{array}{l}\text { More than } 150 \text { numbers of } \\
\text { filled grain per panicle }\end{array}$} \\
\hline \multicolumn{2}{|c|}{ NUMBERS OF PRODUCTIVE TILLERS } \\
\hline $\begin{array}{l}\text { More than average } 4 \\
\text { Numbers of tillers }\end{array}$ & Kaporok, Garomalati, Chikanswarikabar, DhalaBalam, Assam Pisom ,Aaduma \\
\hline
\end{tabular}


Table 5: Details of the microsatellite primers used in present study with their Allele size (bp), Allele diversity, Gene diversity and Polymorphic information content (PIC)

\begin{tabular}{|c|c|c|c|c|c|c|c|c|c|c|c|}
\hline $\begin{array}{l}\text { Sr. } \\
\text { No }\end{array}$ & Primer & Motif & $\begin{array}{l}\mathrm{C} \\
\mathrm{hr}\end{array}$ & $\begin{array}{l}\text { An. } \\
\text { Tmp } \\
\left(c^{0}\right)\end{array}$ & 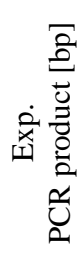 & 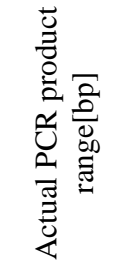 & $\mathrm{Na}$ & $\mathrm{MA}_{\mathrm{fr}}$ & $\mathrm{He}$ & PIC & $\begin{array}{c}\text { Database reference } \\
\text { (Gramene) }\end{array}$ \\
\hline 1 & RM333 & (TAT)19(CTT)19 & 10 & 55 & 191 & $100-200$ & 2 & 0.905 & 0.172 & 0.158 & Tenmykh et al.(2000) \\
\hline 2 & RM482 & (AT)9 & 2 & 55 & 188 & $190-210$ & 3 & 0.667 & 0.470 & 0.394 & \multirow{4}{*}{ Tenmykh et al.(2001) } \\
\hline 3 & RM487 & $(\mathrm{AC}) 10$ & 3 & 55 & 176 & $180-190$ & 2 & 0.828 & 0.285 & 0.245 & \\
\hline 4 & RM491 & (AT) 14 & 12 & 55 & 263 & $280-300$ & 2 & 0.867 & 0.231 & 0.204 & \\
\hline 5 & RM592 & (ATT) 20 & 5 & 55 & 270 & $200-300$ & 4 & 0.744 & 0.419 & 0.388 & \\
\hline 6 & RM175 & $(\mathrm{CCG}) 8$ & 3 & 67 & 95 & $80-180$ & 4 & 0.464 & 0.669 & 0.613 & Tenmykh et al. (2000) \\
\hline 7 & RM205 & (CT)25 & 9 & 55 & 122 & $120-180$ & 3 & 0.696 & 0.469 & 0.423 & \multirow{2}{*}{ Chen et al. (1997) } \\
\hline 8 & RM252 & (CT) 19 & 4 & 55 & 216 & $180-900$ & 6 & 0.300 & 0.777 & 0.743 & \\
\hline 9 & RM276 & (AG)8A3(GA)33 & 6 & 55 & 149 & $150-300$ & 4 & 0.511 & 0.645 & 0.591 & Tenmykh et al.(2000) \\
\hline 10 & RM339 & (CTT)8CCT(CTT) 5 & 8 & 55 & 148 & $120-300$ & 4 & 0.473 & 0.664 & 0.608 & Tenmykh et al.(2000) \\
\hline 11 & RM437 & $(\mathrm{AG}) 13$ & 5 & 55 & 275 & $250-300$ & 3 & 0.643 & 0.513 & 0.450 & \multirow{11}{*}{ Tenmykh et al. (2001) } \\
\hline 12 & RM458 & (TAG) 8 & 8 & 55 & 180 & $180-200$ & 2 & 0.510 & 0.500 & 0.375 & \\
\hline 13 & RM484 & $(\mathrm{AT}) 9$ & 10 & 55 & 299 & $180-300$ & 3 & 0.654 & 0.494 & 0.426 & \\
\hline 14 & RM486 & (CT) 14 & 1 & 55 & 104 & $150-180$ & 2 & 0.754 & 0.371 & 0.302 & \\
\hline 15 & RM488 & (GA)17 & 1 & 55 & 177 & $170-180$ & 2 & 0.655 & 0.452 & 0.350 & \\
\hline 16 & RM505 & (CT) 12 & 7 & 55 & 199 & $200-500$ & 4 & 0.290 & 0.747 & 0.700 & \\
\hline 17 & RM506 & (CT)13 & 8 & 55 & 123 & $100-130$ & 3 & 0.413 & 0.657 & 0.583 & \\
\hline 18 & RM538 & (GA)14 & 5 & 55 & 274 & $280-300$ & 2 & 0.863 & 0.237 & 0.209 & \\
\hline 19 & RM513 & (TC) 11 & 1 & 55 & 262 & $250-260$ & 2 & 0.553 & 0.494 & 0.372 & \\
\hline 20 & RM570 & $(\mathrm{AG}) 15$ & 3 & 55 & 208 & $150-200$ & 3 & 0.455 & 0.619 & 0.539 & \\
\hline 21 & RM566 & (AG)15 & 9 & 55 & 239 & $230-250$ & 3 & 0.558 & 0.582 & 0.512 & \\
\hline 22 & RM167 & (GA) 16 & 11 & 55 & 128 & $120-200$ & 3 & 0.456 & 0.618 & 0.538 & Wu et al. (1993) \\
\hline 23 & RM172 & (AGG)6 & 7 & 55 & 169 & 175 & 1 & 1.000 & 0.000 & 0.000 & Tenmykh et al.(2000) \\
\hline 24 & RM213 & (CT) 17 & 2 & 55 & 139 & $120-200$ & 3 & 0.646 & 0.503 & 0.435 & \multirow{4}{*}{ Chen et al. (1997) } \\
\hline 25 & RM215 & (CT)16 & 9 & 55 & 148 & $120-150$ & 3 & 0.438 & 0.633 & 0.556 & \\
\hline 26 & RM 231 & (CT) 16 & 3 & 55 & 182 & $150-200$ & 3 & 0.527 & 0.594 & 0.518 & \\
\hline 27 & RM257 & $(\mathrm{CT}) 24$ & 9 & 55 & 147 & $140-180$ & 3 & 0.597 & 0.525 & 0.439 & \\
\hline 28 & RM282 & (GA) 15 & 3 & 55 & 136 & $100-150$ & 3 & 0.588 & 0.552 & 0.479 & \multirow{3}{*}{ Tenmykh et al. (2000) } \\
\hline 29 & RM287 & $(\mathrm{GA}) 21$ & 11 & 55 & 118 & $80-120$ & 3 & 0.627 & 0.514 & 0.440 & \\
\hline 30 & RM320 & (AT)11GTAT(GT)13 & 7 & 55 & 167 & $100-400$ & 4 & 0.429 & 0.669 & 0.608 & \\
\hline 31 & RM3 & $(\mathrm{GA}) 2 \mathrm{GG}(\mathrm{GA}) 25$ & 6 & 55 & 145 & $100-150$ & 3 & 0.586 & 0.556 & 0.484 & Panaud et al. (1996) \\
\hline 32 & RM30 & (AG)9A(GA)12 & 6 & 55 & 105 & $70-100$ & 3 & 0.542 & 0.577 & 0.498 & Chen et al. (1997) \\
\hline 33 & RM52 & (AG)19 & 8 & 55 & 240 & $250-500$ & 3 & 0.867 & 0.239 & 0.225 & Tenmykh et al. (2000) \\
\hline 34 & RM146 & (CT)11-(CT)7 & 5 & 55 & 345 & $300-350$ & 3 & 0.607 & 0.503 & 0.407 & Tenmykh et al. (2000) \\
\hline 35 & RM233 & (CT)20 & 2 & 55 & 162 & $150-170$ & 2 & 0.558 & 0.493 & 0.372 & Chen et al. (1997) \\
\hline 36 & RM254 & (TC)6ATT(CT)11 & 11 & 55 & 165 & $100-800$ & 5 & 0.453 & 0.687 & 0.630 & Chen et al. (1997) \\
\hline 37 & RM285 & $(\mathrm{GA}) 12$ & 9 & 55 & 205 & $200-800$ & 4 & 0.444 & 0.697 & 0.648 & Tenmykh et al. (2000) \\
\hline 38 & RM315 & (AT)4(GT)10 & 1 & 55 & 133 & $100-130$ & 3 & 0.623 & 0.523 & 0.453 & Tenmykh et al. (2000) \\
\hline 39 & RM452 & (GTC)9 & 2 & 55 & 209 & $200-230$ & 2 & 0.964 & 0.069 & 0.067 & Tenmykh et al. (2001) \\
\hline 40 & RM 276 & (AG)8A3(GA)33 & 6 & 55 & 149 & $150-180$ & 2 & 0.600 & 0.480 & 0.365 & Tenmykh et al. (2000) \\
\hline \multicolumn{2}{|c|}{ TOTAL } & & & & & & 119 & 24.35 & 19.89 & 17.34 & \\
\hline \multicolumn{2}{|c|}{ AVERAGE } & & & & & & 2.97 & 0.608 & 0.497 & 0.433 & \\
\hline
\end{tabular}

Chr.: Chromosome location, An. Tmp: Annealing temperature, Exp.PCR product [bp]: Expected PCR product size in base pair, Na: Total number of alleles per locus, $\mathrm{MA}_{\mathrm{fr}}$ : Major allele frequency, He: Gene Heterozygosity, PIC: Polymorphism information content 
Sujan Acharjee et al.

Table 6: Similarities in the grouping pattern of landraces under mean value analysis and their clustering pattern under molecular analysis

\begin{tabular}{|c|c|c|}
\hline Traits & \multicolumn{2}{|l|}{ Molecular Clustering pattern } \\
\hline & Name of the landraces & Sub group \\
\hline \multirow[b]{2}{*}{$\begin{array}{l}\text { Root length } \\
\text { (more than } 20 \mathrm{~cm} . \text { ) }\end{array}$} & Kaporok, Releng, Tarkol, Tarikol Kolte & $\mathrm{B}_{1}$ \\
\hline & $\begin{array}{l}\text { Maimi Hungar,Chikanswari Kabar } \\
\text { Madoop,Garomalati }\end{array}$ & $\begin{array}{l}\text { Scattered in } \\
\text { different } \\
\text { cluster }\end{array}$ \\
\hline \multirow{3}{*}{$\begin{array}{l}\text { Root: Culm ratio } \\
\text { (more then } 0.3 \text { ) }\end{array}$} & Kaporok, Releng, Tarkol, Tarikol Kolte & $\mathrm{B}_{1}$ \\
\hline & Madoop, Maimi Watoklok & $\mathrm{A}_{1}$ \\
\hline & MaimiHungar & $\mathrm{C}_{1} \mathrm{C}$ \\
\hline \multirow[t]{2}{*}{$\begin{array}{l}\text { Moderate to high leaf } \\
\text { drying index }\end{array}$} & Chinal, Sada Biroin, Kala Jira, Kanchali & $\begin{array}{l}\text { Scattered in } \\
\text { different } \\
\text { cluster }\end{array}$ \\
\hline & Yang Dhan, Badaya & $\mathrm{C}_{2} \mathrm{~A}$ \\
\hline $\begin{array}{l}\text { Low to moderate leaf } \\
\text { rolling index }\end{array}$ & Kaporok, Releng, Tarkol, Beti & $\mathrm{B}_{1}$ \\
\hline $\begin{array}{l}\text { Moderate to high leaf } \\
\text { drying index }\end{array}$ & Badaya, Yang Dhan & $\mathrm{C}_{2} \mathrm{~A}$ \\
\hline $\begin{array}{l}\text { Plant height } \\
\text { (more than } 125 \mathrm{~cm} . \text { ) }\end{array}$ & Chikanswari Kabar & $\mathrm{C}_{2} \mathrm{C}$ \\
\hline $\begin{array}{l}\text { More than average } 4 \\
\text { numbers of productive } \\
\text { tillers }\end{array}$ & $\begin{array}{l}\text { Kaporok, Garomalati, Chikanswari Kabar, } \\
\text { Dhala Balam, Assam Paisom, Aaduma }\end{array}$ & $\begin{array}{l}\text { Scattered in } \\
\text { different } \\
\text { cluster }\end{array}$ \\
\hline $\begin{array}{l}100 \text { seed weight } \\
\text { (more than } 2.5 \mathrm{gm} . \text { ) }\end{array}$ & $\begin{array}{l}\text { Maimi Hungar, Gaigash, Maimi Watoklok, } \\
\text { Maimi Red, Bangbu Jhum, Releng, Galong, } \\
\text { Garomalati, Fazu Sen, BetiKalai, Chikanswari } \\
\text { Kabar, Lal Biroin, DhalaBalam, Tarikol } \\
\text { Kolte, Tarkol, Waibang, Jhum Bini, Bongbu, } \\
\text { Kaladhan, Turkey, Badaya }\end{array}$ & $\begin{array}{l}\text { Scattered in } \\
\text { different } \\
\text { cluster }\end{array}$ \\
\hline $\begin{array}{l}\text { Numbers of filled grain } \\
\text { per panicle (more than } 150 \\
\text { Numbers) }\end{array}$ & $\begin{array}{l}\text { Galong, Garomalati, ChikanswariKabar, } \\
\text { Madoop, Tarkol }\end{array}$ & $\begin{array}{l}\text { Scattered in } \\
\text { different } \\
\text { cluster }\end{array}$ \\
\hline $\begin{array}{l}\text { Grain yield per plant } \\
\text { (more than } 10 \mathrm{gm} \text { ) }\end{array}$ & $\begin{array}{l}\text { ChikanswariKabar, Garomalati, Galong, } \\
\text { Turkey, Tarkol, Dhalabalam, MaimiHungar }\end{array}$ & $\begin{array}{l}\text { No specific } \\
\text { cluster }\end{array}$ \\
\hline
\end{tabular}


Marker based genetic variability analysis of rice (Oryza sativa L.) landraces for drought tolerance

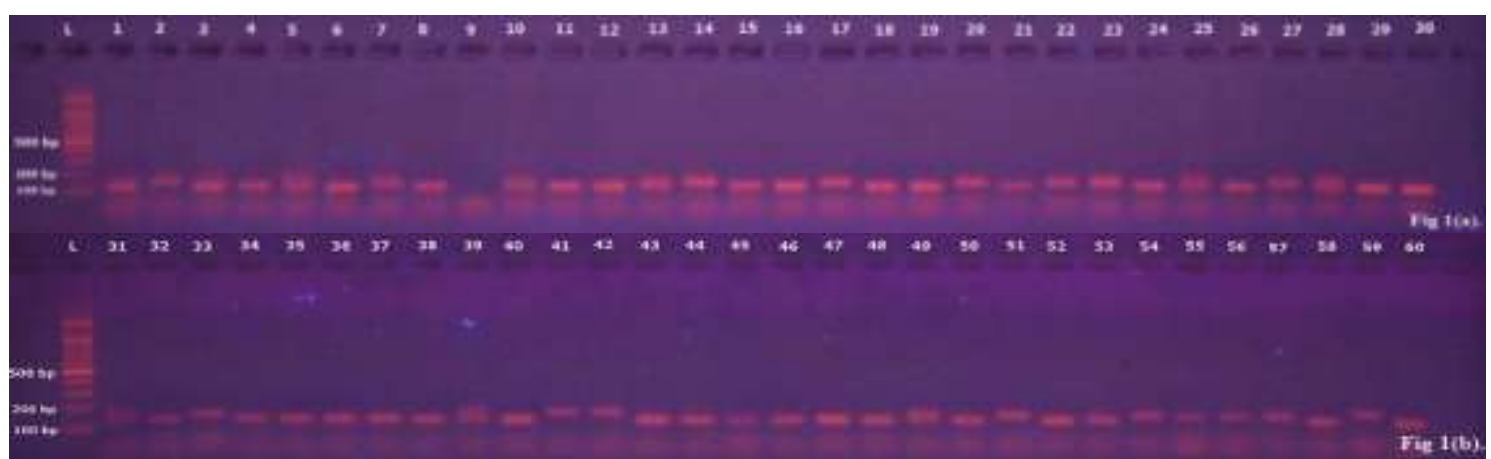

Fig. 1 a \& b: Amplification profile of 60 rice landraces generated through microsatellite primer - RM 257

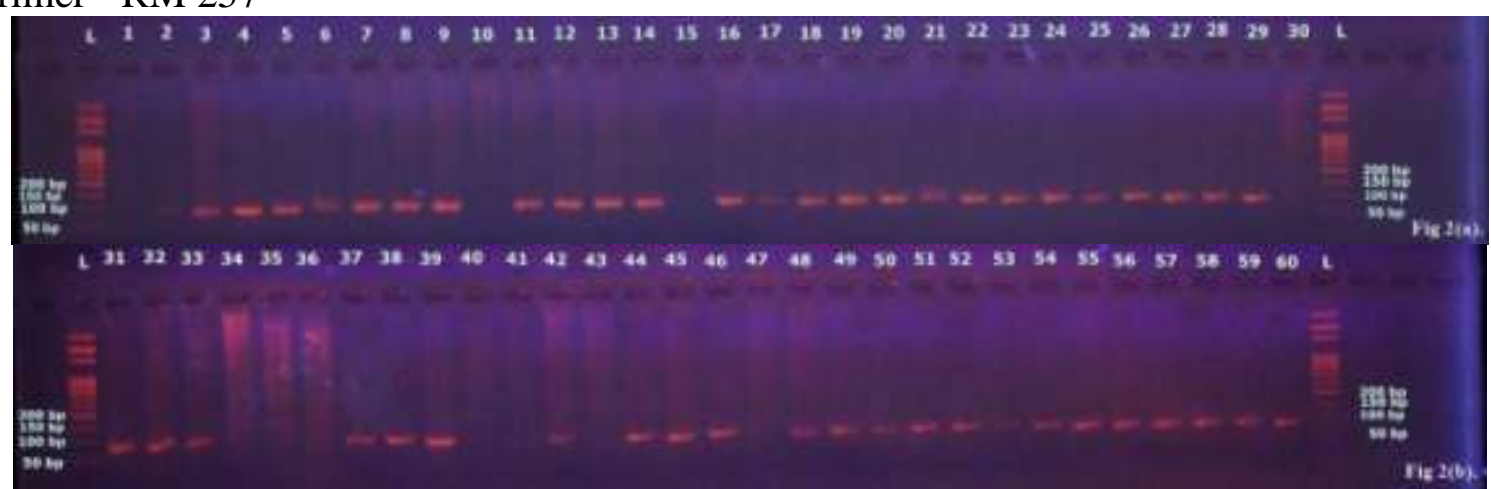

Fig. 2 a \& b: Amplification profile of 60 rice landraces generated through microsatellite primer - RM 538

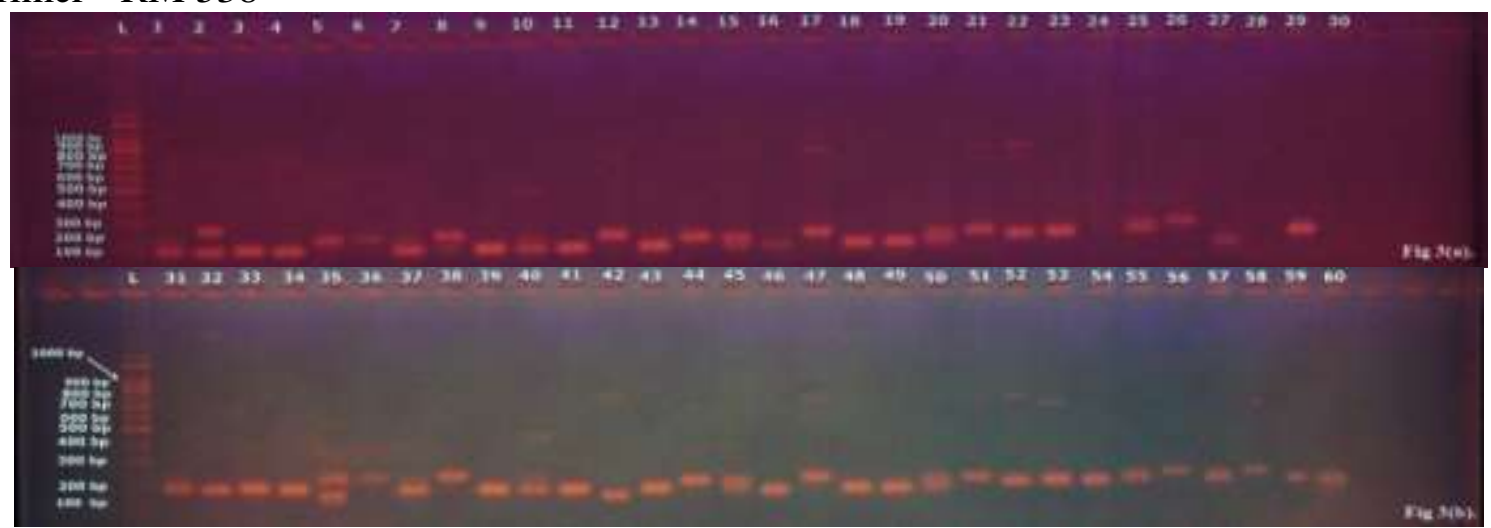

Fig. 3 a \& b: Amplification profile of 60 rice landraces generated through microsatellite primer - RM 254

L- DNA ladder, 1. Kaporok 2. Releng 3. Beti 4. MaimiUzra 5. Kalikhasa 6. Chinal 7. American 8. KhasaKasam 9. Biroin10.Galong 11. FazuVom 12. Garo Malati 13. Maimi Usha 14. Maimi Red 15. Suri 16.Lebuka 17.Aaduma 18.Fazu Sen 19.Fazu N'G 20.Beti Kalai 21.Saanki ka Phool 22.Bihar 23.Chikanswari Kabar 24.Bangbu Jhum 25.Lal Biroin 26.Dhala Balam 27.Goria 28.Bahadur 29.M.W.Mandoori 30.Mami Hungar 31.Darka Sona 32.Tarikol Kolte 33.Sada Biroin 34.Maimi Taukha 35.Saluma 36.Tarkol 37.Madoop 38.Waibang 39.Jhum Bini 40.Fazu Sen (White) 41.Bongbu 42.Sadok 43.Kala Jira 44.Gaigash 45.Vanbang 46.Makajaria 47.Jilong 48.AmericanRation49.KalaDhan 50.Turkey 51.Saanki Kachak 52. Maimi Ukhlao 53. Maiwasha 54. MaimiWatolok 55. SantinWakhum 56. YangDhan 57. Badaya 58. Kanchali 59. Australian Biroin 60. Assam Paisom 
Sujan Acharjee et al.

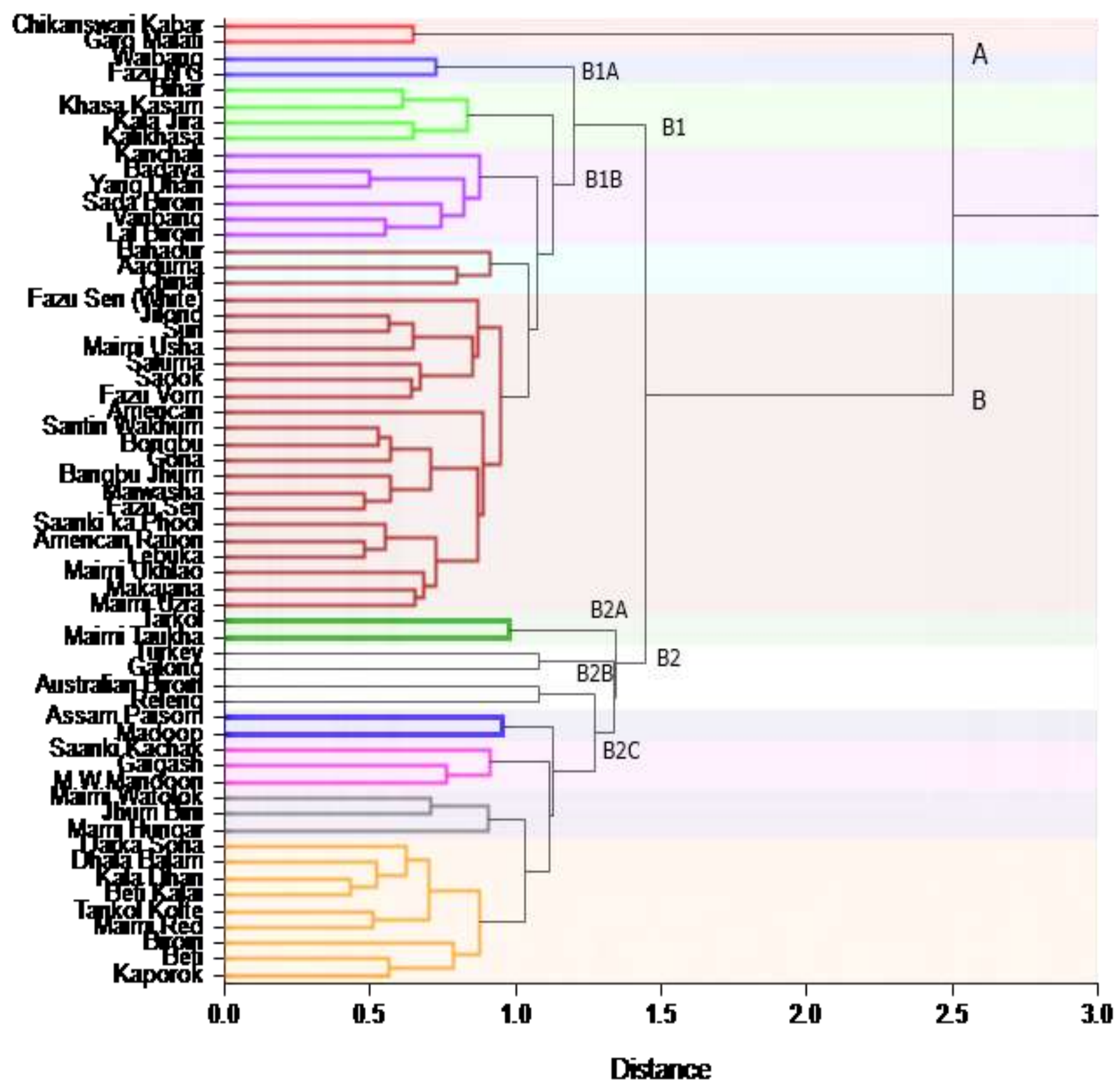

Fig 4 Clustering pattern of 60 nos. of rice landraces under morphological analysis 


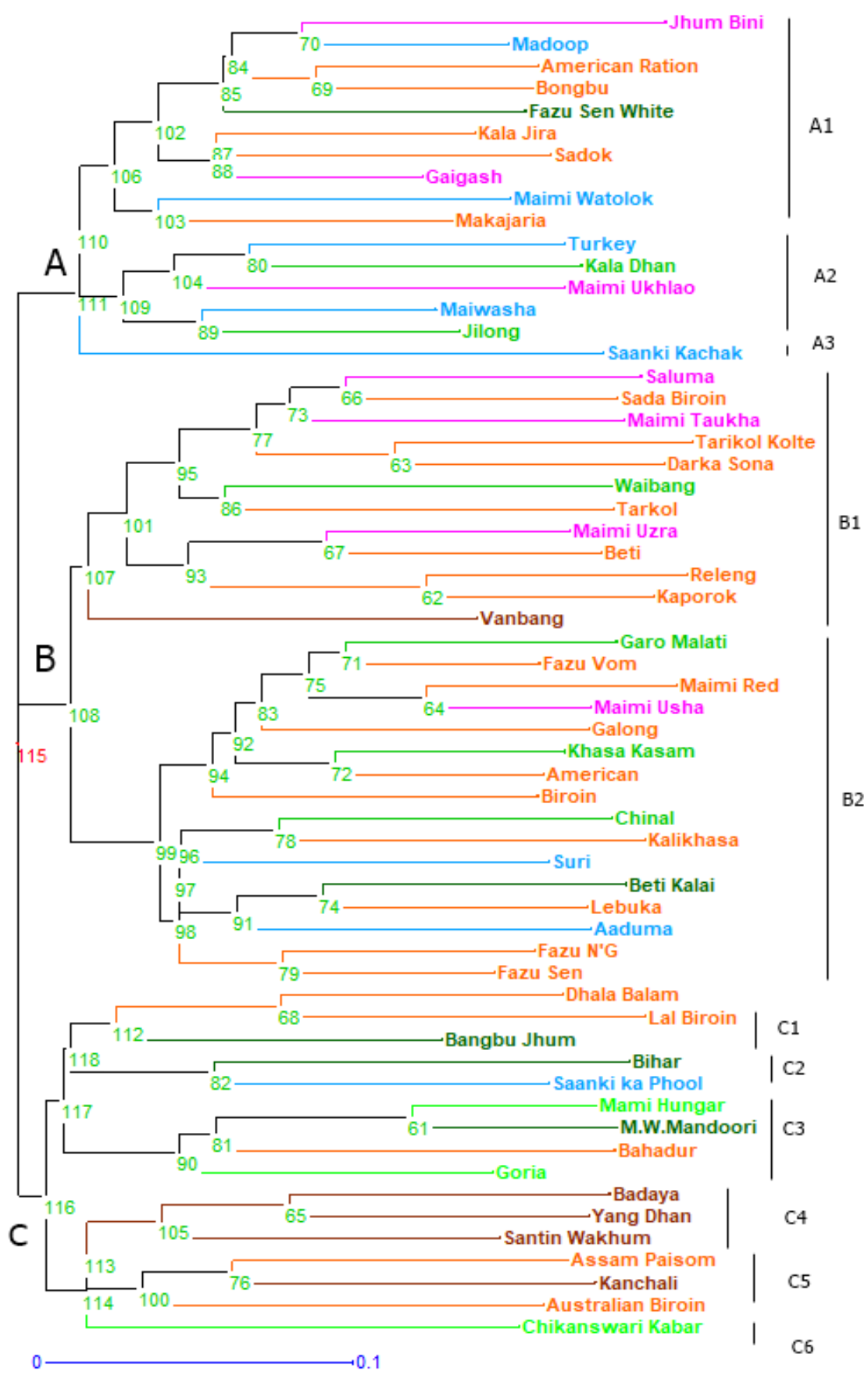

Fig 5 Clustering pattern of 60 nos. of rice landraces under molecular analysis 


\author{
e- ISSN 2314-5501 (online) \\ E.mail: aasdjournal@yahoo.com
}

\section{EFERENCES}

Acharjee, S.; Chakraborty. N.R. and Das, S.P. (2019). Exploration and agromorphological evaluation of rice (Oryza sativa L.) land races grown under theFukagawa, N.K. and Ziska, L.H. (2019). Rice: upland ecosystem of Tripura. J. Pharmacognosy Phytochem., 8: 2316-2323. disequilibrium analysis of chickpea (Cicer arietinum L.) genotypes using genomewide DArTseq-based SNP markers. Genes, 10:676. Importance for Global Nutrition. J. Notr. Sci. Vitaminol., 65: S2-S3.

Adak, S.; Datta, S.; Bhattacharya, S.; Ghose, T.K. Gramene Microsat. Available at <https: and Majumder, A.L. (2020). Diversity //archive.gramene.org/markers/microsat/al analysis of selected rice landraces from 1-ssr.html.> Accessed on 16 August 2018. West Bengal and their linked molecularHaider, Z.; Khan, A.S. and Zia, S. (2012). markers for salinity tolerance. Physiol. Mol. Biol. Plants, 26 :669-682.

Anupam. A.; Imam, J.; Quatadah, S.M.; Siddaiah, A.; Das, S.P.; Variar, M. and Mandal, N.P. (2017). Genetic diversity Correlation and path coefficient analysis of yield components in rice (Oryza sativa L.) under simulated drought stress condition. American-Eurasian J. Agric. Environ. Sci., 12: 100-104. analysis of rice germplasm in TripuraHamidah, M.S.; Mohd, Y.R.; Asfaliza, R.; Yusuff, State of Northeast India using drought and blast linked markers. Rice Sci., 24: 10-20. O.; Hanafi, M.M.; Harun, A.R.; Zakiah. M.Z. and Samuel, C.C. (2020). Genetic Becerra, V.; Paredes, M.; Gutiérrez, E. and Rojo, C. (2015). Genetic diversity, identification, and certification of Chilean rice varieties using molecular markers. Chilean J. Agric. Res., 75:267-274.

\section{IRRI}

Botstein, D.; White, R.L.; Skolnick, M. and Davis, R.W. (1980). Construction of a genetic linkage map in man usingJan, S.J.K. :PIC calculator (2002). Available at < restriction fragment length polymorphism. Am. J. Hum. Gen., 32: 314- 331. Johnson, H.W.; Robinson, H.F. and Comstock, R.

Chen, X.; Temnykh, S.; Xu, Y.; Cho, Y.G. and McCouch, S.R. (1997). Development of a microsatellite framework map providing diversity and variability among pigmented rice germplasm using molecular marker and morphological traits. Biotechnol. Biotechnolog. Equip., 34(1): 747-762. genome-wide coverage in rice (OryzaKumar, S.; Singh, N.K.; Kumar, R. and Kumar, C. sativa L.). Theoretical Appl. Gen., 95: 553-567.

DeWoody, J.A.; Honeycutt, R.L. and Skow, L.C. (1995). Microsatellite markers in whitetailed deer. J.Heredity, 86: 317-319.

Luo, X.; Tan, Y.; Ma, C.; Tu, J.; Shen, J.; Yi, B.

Eid, M. (2019). RAPD fingerprinting and genetic relationships of some wheat genotypes. Int. J. Gen. Genom., 7:1

Farahani, S.; Maleki, M.; Mehrabi, R.; Kanouni, H.; Scheben, A.; Batley, J. and Talebi, R. (2019). Whole genome diversity, population structure, and linkage (1955). Estimates of genetic and environmental variability in soybeans. Agronomy J., 47: 314-318.

(2015). Genetic divergence analysis for morpho-physiological traits of rice (Oryza sativa L.) under drought condition. Ecoscan, 9: 653-658. and $\mathrm{Fu}, \mathrm{T}$. (2019). Highthroughput identification of SNPs reveals extensive heterosis with intra-group hybridization and genetic characteristics in a large rapeseed (Brassica napus L.) panel. Euphytica, 215:157. 


\section{Marker based genetic variability analysis of rice (Oryza sativa L.) landraces for drought tolerance}

Melaku, G.; Zhang, S. and Haileselassie, T. (2018). Comparative Evaluation of Rice SSR Markers on Different Oryza Species. J. Rice Res. Develop., 1(1):38-48.
Perrier, X. and Jacquemoud-Collet, J.P. (2006). DARwin software. Available at <http: //darwin. cirad.fr/Darwin.> Accessed on September 19,2019.

Murray, M.G. and Thompson, W.F. (1980). RapidPerween, S.; Kumar, A.; Singh, S.; Satyendra, isolation of high molecular weight plant K.M. and Ranjan, R. (2020). Genetic DNA. Nucleic Acids Res., 8:4321-4325.

Nachimuthu, V.V.; Muthurajan, R.; Duraialaguraja, S.; Sivakami, R.; Pandian, B.A.; Ponniah, G. et al. (2015) Analysis of Population Structure and Genetic Variability Parameters for Yield and Yield Diversity in Rice Germplasm Using SSRPradhanm A.K.; Kalita, J.; Lahkar, L.; Gurung, Markers: An Initiative Towards Association Mapping of Agronomic Traits in Oryza Sativa. Rice, 8: 30.

Nagy, S.; Poczai, P.; Cernak, I.; Gorji, A.M.; Hegedus, G. and Taller, J. (2012). PICcalc: An Online Program to Calculate Polymorphic Information Content for Molecular Genetic Studies. Biochem. Gen., 50: 670-672.

Related Traits in Rice (Oryza sativa L.) under Irrigated and Drought Stress Condition. Int. J. Curr. Microbiol. Appl. Sci., 9: 1137-1143.

L.; Ghritlahre, S.K. and Tanti, B. (2020). Stress Management in Crops by Utilizing Landraces: Genetics and Plant Breeding Perspective. In: Roycho-wdhury, R.; Choudhury, S.; Hasanuzzaman, M. and Srivastava, S. (eds) Sustainable Agriculture in the Era of Climate Change. Springer, Cham., p.1-21

Ramadan, E.A.; Elmoghazy, A.M. and ElNgangkham, U.; Dash, S.; Parida, M.; Mowafi. H.F. (2015). Molecular markers Samantaray, S.; Nongthombam, D.; based genetic diversity analysis for Yadav, M.K.; et al. (2019). The drought tolerance in rice (Oryza Sativa L.) potentiality of rice microsatellite markers using SSR Markers. Int. J. Sci. Res. in assessment of cross-species Agric. Sci., 2:137-146.

transferability and genetic diversity of rice Rasheed, A.; Hassan, M.U.; Aamer, M.; Bian, and its wild relatives. Biotech., 9:217. J.M.; Xu, Z.R.; He, X.F.;Yan, G. and Wu, Nithya, N.; Beena, R.; Roy, S.; Abida, P.S.; Jayalekshmi, V.G.; Viji, M.M. and Manju, R.V. (2020). Genetic Variability, Heritability, Correlation Coefficient and Z.M. (2020). Iron toxicity, tolerance and quantitative trait loci mapping in rice; a review. Appl. Ecol. Environ. Res., 18:7483-7498.

Path Analysis of Morpho-physiological Ravikiran, K.T.; Krishnamurthy, S.L.; Warraich, and Yield Related Traits of Rice under Drought Stress. Chem. Sci. Rev. Letters, 9: 48-54.

Panaud, O.; Chen, X. and McCouch, S.R. (1996). Development of microsatellite markers A.S. and Sharma, P.C. (2018). Diversity and haplotypes of rice genotypes for seedling stage salinity tolerance analyzed through morpho-physiological and SSR markers. Field Crops Res., 220: 10-18.

and characterization of simple sequenceStandard Evaluation System for Rice (2002) length polymorphism (SSLP) in rice International Rice Research Institute, (Oryza sativa L.). Mol. General Gen., 252: 597-607.

Manila.

http://www.knowledgebank.irri.org/image s/docs/rice-standard-evaluation-

Panja, S.; Garg, H.; Mandi, V.; Sarkar, K. and Bhattacharya, C. (2017). Effect of water stress at tillering stage on different system.pdf. Accessed on February $\underline{22,2015}$. morphological traits of rice (Oryza sativa Temnykh, S.; DeClerck, G.; Lukashova, A.; 1) genotypes. Int. J. Agric. Sci. Res., 7: 471-480.

Lipovich, L.; Cartinhour, S. and McCouch, S. (2001). Computational and experimental analysis of microsatellites in 
Sujan Acharjee et al.

rice (Oryza sativa L.): frequency, length variation, transposon associations, and genetic marker potential. Genome Res., 11: $1441-1452$.

Yadav, S.; Singh, A.; Singh, M.R.; Goel. N.; Temnykh, S.; Park, W.D.; Ayres, N.M.; Vinod, K.K.; Mohapatra, T. and Singh, Cartinhour, S.; Hauck, N.; Lipovich, L.; A.K. (2013). Assessment of genetic Cho, Y.G.; Ishii, T. and McCouch, S.R. (2000). Mapping and genome organization of microsatellite sequences in rice (Oryza diversity in Indian rice germplasm (Oryza sativa L.): Use of random versus traitlinked microsatellite markers. J. Genetics. sativa L.). Theoretical Appl. Gen., 100: 92: 697-712.

Wu, K.S. and Tanksley, S.D. (1993). Abundance, polymorphism and genetic mapping of microsatellites in rice. Mol. General Gen., 241: 225-235. 